\title{
A note on platelet adhesiveness in ischaemic heart disease
}

\author{
P. J. NESTEL \\ From the Department of Medicine, University of Melbourne
}

SYNOPSIS Platelet adhesiveness was measured in vitro in a group of patients with ischaemic heart disease and in a group of matched healthy subjects using either $1 \%$ EDTA or $3.8 \%$ sodium citrate as anticoagulant. When sodium citrate was used platelet adhesiveness was significantly greater in the group with ischaemic heart disease. However, no difference was found when EDTA was used.

In both groups platelet adhesiveness was found to be significantly greater with sodium citrate than with EDTA.

A property of blood platelets is adhesiveness which is an important mechanism in normal haemostasis, since plugs of adherent platelets are found at the earliest stages in the formation of a white thrombus (Zucker, 1947). A tendency towards increased adhesiveness has therefore been sought in conditions characterized by pathological thrombosis. Increased platelet adhesiveness has been demonstrated by Wright (1942) postoperatively and post partum and by Moolten, Vroman, Vroman, and Goodman (1949) in a variety of thromboembolic diseases, including acute myocardial infarction. More recently McDonald and Edgill (1959) have found increased adhesiveness in angina pectoris, acute coronary insufficiency, and a recent myocardial infarction. Since the increasing incidence of ischaemic heart disease in highly industrialized white populations is said to be related to an increased predisposition towards arterial thrombosis (Morris, 1951), a further study of platelet adhesiveness in ischaemic heart disease seemed warranted.

The methods used for measuring platelet adhesiveness have not been found universally satisfactory and a variety of techniques have been described. A recent method in which adhesiveness is measured in platelet-rich plasma rather than in whole blood (Hutchison, Stark, and Chapman, 1959) has been employed in the present study.

\section{MATERIALS AND METHODS}

Two groups of male subjects who were matched for age were investigated.

Group 1 comprised 13 patients who had suffered a

Received for publication 30 September 1960. myocardial infarction at least four months previously and were well at the time of the investigation. They were not receiving treatment. Their mean age was 54 years.

Group 2 comprised 10 healthy subjects whose mean age was 52 years.

Blood was collected from subjects after an overnight fast with siliconed glassware into siliconed tubes kept chilled at $4^{\circ} \mathrm{C}$. A two-syringe technique was used and the blood in the first syringe discarded. Duplicate samples were collected into tubes which contained either $3.8 \%$ sodium citrate or $1 \%$ EDTA, $9 \mathrm{ml}$. blood being added to $1 \mathrm{ml}$. anticoagulant. Platelet-rich plasma was obtained be spinning blood at 1,000 r.p.m. for 15 minutes at $4^{\circ} \mathrm{C}$. Platelet adhesiveness was measured within 30 minutes. Aliquots, each of $3 \mathrm{ml}$., of the plasmas were rotated on a turntable at 33 r.p.m. at an angle of $45^{\circ}$ in a refrigerator, since platelets are adhesive in citrated plasma only at low temperatures (Hellem, 1960). Samples were taken for platelet counts at a standard level in the tubes at the beginning and at half-hour intervals. The time taken for the platelet count to fall to half its initial value was taken as a measure of adhesiveness when citrate was used as anticoagulant. Since adhesiveness is greatly decreased when EDTA is used (Hellem, 1960), the percentage fall after two hours was taken as the index of adhesiveness when EDTA was used as anticoagulant. Platelets were counted directly. If more than two platelets were clumped together they were not counted.

\section{RESULTS}

The rates of fall in the number of platelets were on the whole linear over the period of observation.

Table I shows the times taken for the platelet counts to fall to half their initial values with citrate as anticoagulant and the percentage falls in platelet counts at two hours with EDTA as anticoagulant. 
TABLE I

COMPARISON OF FALLS IN PLATELET COUNTS IN PATIENTS WITH MYOCARDIAL INFARCTION AND IN CONTROLS USING $3.8 \%$ SODIUM CITRATE OR $1 \%$ EDTA AS ANTICOAGULANTS

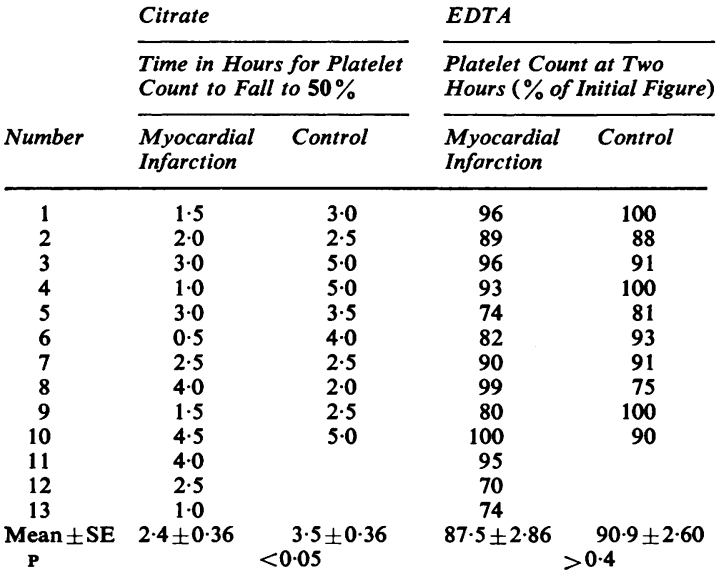

The individual figures for all subjects and the mean values for the two groups are shown. Using citrate, the mean values and standard errors were $2 \cdot 4 \pm 0 \cdot 36$ hours for the infarction group and $3.5 \pm 0.36$ hours for the control group. This difference was statistically significant $(P=<0 \cdot 05)$. Using EDTA, the mean percentage fall at two hours was to $87 \cdot 5 \pm 2.86 \%$ of the initial figure for the infarction group and to $90.9 \pm$ $2.60 \%$ for the control group. This difference was not significant.

To determine the effect in vitro of the two anticoagulants on platelet adhesiveness in general, the two-hour platelet counts in all 23 subjects were calculated as a percentage fall. This was done for citrate and EDTA. The mean fall was to $63 \%$ of the initial figure with citrate and to $89 \%$ with EDTA, and this difference was statistically significant $(P=<0.001)$. Platelet adhesiveness was therefore greater when citrate was used.

At the completion of each experiment a sample of plasma was obtained with a Pasteur pipette from the bottom of the tube and examined microscopically. Whenever citrate was used many clumps consisting of large numbers of platelets were seen. With EDTA very few clumps consisting only of a few platelets were found.

\section{DISCUSSION}

When citrate was used as an anticoagulant for the collection of blood, the platelets of a group of patients with a myocardial infarction were found to adhere significantly more than the platelets of healthy subjects matched for age. This is in agreement with the findings of McDonald and Edgill (1959) who investigated a group of patients with a more recent infarction than in the present group. McDonald and Edgill did state, however, that even at a later stage the platelets of such patients remained abnormally adhesive. However, when EDTA was used as anticoagulant in the present experiments no such difference was found.

The interpretation of these results is difficult since the mechanism of platelet adhesiveness has not been entirely established. Hellem (1960) has demonstrated the influence of red blood cells on adhesiveness. He showed that a substance, named factor $\mathbf{R}$, was released from red cells, and that in the presence of factor $R$ the non-adhesive platelet in citrated platelet-rich plasma became adhesive. This, however, does not explain the findings that platelets which are not adhesive in citrated plasma at room temperature become adhesive at low temperatures and that platelets suspended in saline are adhesive even at room temperature (Hellem, 1960). It is possible that the increased adhesiveness of platelets at low temperatures is related to the morphological alterations which have been found to occur when platelets are stored at $4^{\circ} \mathrm{C}$. (Zucker and Borrelli, 1954).

The mechanisms responsible for the difference found between the two groups with citrated plateletrich plasmas is therefore obscure. In addition aggregation of platelets contributed to the falls in platelet counts. However, Hellem (1960) considers that the mechanisms responsible for aggregation and adhesiveness are related, since he found that factor $\mathbf{R}$ produced platelet adhesiveness in low concentration and aggregation in high concentration. The finding of similar platelet adhesiveness in the two groups when EDTA was used as anticoagulant is probably of little significance, since very little adhesiveness could be demonstrated with this anticoagulant. This is in agreement with Hellem (1960) and may also be related to an alteration in platelet morphology (Zucker and Borrelli, 1954).

\section{REFERENCES}

Hellem, A. J. (1960). Scand. J. Lab. clin. Invest., 12, Suppl. 51. Hutchison, H. E. Stark, J. M., and Chapman, J. A. (1959). J. clin. path., 12, 265.

McDonald, L., and Edgill, M. (1959). Lancet, 1, 1115.

Moolten, S. E., Vroman, L., Vroman, G. M. S., and Goodman, B. (1949). Arch. Intern. Med., 84, 667.

Morris, J. N. (1951). Lancet, 1, 69.

Wright, H. Payling (1942). J. Path. Bact., 54, 461.

Zucker, M. B. (1947). Amer. J. Physiol., 148, 275.

, and Borrelli, J. (1954). Blood, 9, 602. 